\title{
Tangence
}

\section{Enfants, adolescents, adultes : mutations de l'horizon d'attente et de l'intentionnalité dans l'oeuvre littéraire de Dominique Demers}

\section{Daniel Chouinard}

Numéro 67, automne 2001

L'écriture pour la jeunesse : de la production à la réception

URI : https://id.erudit.org/iderudit/009620ar

DOI : https://doi.org/10.7202/009620ar

Aller au sommaire du numéro

Éditeur(s)

Presses de l'Université du Québec

ISSN

0226-9554 (imprimé)

1710-0305 (numérique)

Découvrir la revue

Citer cet article

Chouinard, D. (2001). Enfants, adolescents, adultes : mutations de l'horizon d'attente et de l'intentionnalité dans l'oeuvre littéraire de Dominique Demers. Tangence, (67), 127-137. https://doi.org/10.7202/009620ar 


\title{
Enfants, adolescents, adultes : mutations de l'horizon d'attente et de l'intentionnalité dans l'œuvre littéraire de Dominique Demers.
}

\author{
Daniel Chouinard, University of Guelph
}

Jusqu'à récemment, c'est-à-dire de ses débuts en 1991 au tournant de 1997, le projet littéraire de Dominique Demers s'articulait à partir d'une distinction des plus claires quant au public visé : pour les enfants, des récits humoristiques suscitant le goût de la lecture; pour les adolescents, des romans volontiers déchirants et dramatiques liés à une crise d'identité et à l'apprentissage de la vie. Position qu'elle a réitérée à maintes reprises: entre autres exemples, dans une notice biographique sur Internet, antérieure à 1997 et disparue depuis: "J'écris [...] des romans drôles pour enfants et des romans tristes pour adolescents ${ }^{1}$.

Toutefois, depuis la parution en un volume pour grand public de Marie-Tempête et de Maina ${ }^{3}$ en 1997, et surtout, avec le succès de son premier roman pour adultes, Le pari, la distincfion qui prévalait à l'origine s'est quelque peu embrouillée. Cette réorientation partielle ou cette nouvelle polyvalence dans la carrière de Dominique Demers soulève la problématique du double lectorat des ouvres de la littérature pour la jeunesse. Non pas célle de la complicité entre parents lecteurs et enfants en situation

1. Voir le site suivant: http:/www.maclean-hunter.qc.ca/pages_journalistes/ demers.html

2. Dominique Demers, Marie-Tempête, Montréal, Québec/Amérique, 1997. Désormais les références à ce roman seront indiquées par le sigle $M T$, suivi de la page, et placées entre parenthèses dans le corps du texte.

3. Dominique Demers, Maina, t. 1: L'appel des loups, t. 2: Le pays de Natak, Montréal, Québec/Amérique Jeunesse, coll. "Titan +»1997; désormais les références à ces romans seront indiquées respectivement par les sigles $A L$ et $P N$, suivis de la page, et placées entre parenthèses dans le corps du texte. Dominique Demers, Maina (en un volume), Montréal, Québec/Amérique, 1997; désormais, les références à ce roman seront indiquées par le sigle $M A$, suivi de la page, et placées entre parenthèses dans le corps du texte.

4. Dominique Demers, Le pari, Montréal, Québec/Amérique, 1999. Désormais, les références à ce roman seront indiquées respectivement par le sigles $L P$, suivi de la page, et placées entre parenthèses dans le corps du texte. 
d'apprentissage de la lecture et de la culture, déjà perceptible dans les contes des $\mathrm{XVII}^{\mathrm{e}}$ et $\mathrm{XVIII}^{\mathrm{e}}$ siècles ou observable dans la réécriture des chefs-d'œuvre pour lecteurs enfants et préadolescents, mais bien celle de la zone grise qu'évoque Danielle Thaler à propos des collections spécialisées pour les plus de douze ans que fréquentent sans complexe de nombreux adultes ${ }^{5}$. Pour cette dernière catégorie de lecteurs partagée entre adolescents et adultes, la problématique s'énonce à la fois comme celle d'une double réception (le consommateur réel/le lecteur comme construction du système narratif) et celle d'un double lectorat (le public adolescent ciblé/tous les autres lecteurs). Il se produit en effet une transformation interne et externe des signes littéraires, comme le montrent les cas de censure, tout comme se manifestent de nouvelles orientations, strictement éditoriales, imposées de l'extérieur et qui, elles aussi, influent sur la perception de l'œuvre.

Se référer à la trilogie "Marie-Lune" ou au volume "MarieTempête "implique forcément un changement dans l'horizon d'attente ${ }^{6}$. Pour des raisons de clarté stylistique, nous désignerons du nom de l'héroïne, Marie-Lune, la série des trois romans destinés expressément au lectorat adolescent, et nous conserverons pour le texte paru à l'intention des adultes, le surnom paratextuel, Marie-Tempête, ou le titre de la version pour adultes, MarieTempête. Rappelons par ailleurs que la quatrième de couverture indique, pour chacun des volumes de la trilogie et dans un cercle rouge, "Marie-Lune $1^{\text {re }} 2^{\text {e }}$ et $3^{e}$ ". Avant de spéculer sur les motivations économiques, idéologiques et esthétiques que sous-tendent

5. Danielle Thaler, "Littérature de jeunesse : un concept problématique ", Canadian Cbildren's Literature/Littérature canadienne pour la jeunesse (CCL/LCJ), Guelph (Ontario), n' 83, automne 1986, vol. 23, n'3, p. 26-38. Comme le rappelle l'auteure, *[I]es romans pour adolescents, souvent empruntés à la littérature populaire (pour adultes) ou dont l'écriture les en rapproche fort, ont plus d'affinités avec les genres et les romans pour adultes qu'ils n'en ont avec les romans pour enfants* (p. 28).

6. Dominique Demers, Un biver de tourmente, Montréal, La courte échelle, coll. "Roman + ", 1992. Désormais les références à ce roman seront indiquées par le sigle $H T$, suivi de la page, et placées entre parenthèses dans le corps du texte. Dominique Demers, Les grands sapins ne meurent pas ("Marie-Lune II "), Montréal, Québec/Amérique Jeunesse, coll. "Titan", 1993. Dominique Demers, Ils dansent dans la tempête ("Marie-Lune III"), Montréal, Québec/Amérique Jeunesse, coll. "Titan", 1994. Le premier roman, republié chez Québec/Amérique Jeunesse, dans la coll. "Titan ", devient "Marie-Lune I". 
de telles modifications ou d'évoquer un modèle culturel perceptible chez plusieurs autres écrivains du Québec et lié à l'institution littéraire, tentons d'observer de plus près la double articulation de la lecture.

\section{Les impératifs de la mise en marché}

Tout d'abord, Le pari, où chaque élément de la présentation matérielle de l'ouvrage sursignifie "roman pour adultes "ou accentue la dichotomie jeunesse/adulte: le format $(15 \mathrm{~cm}$ sur $22 \mathrm{~cm}$, rarissime pour les œuvres québécoises destinées à la jeunesse); la couverture: un photomontage d'un paysage maritime d'été - "un roman d'orages et d'embellies. ( $L P$, quatrième de couverture) - sur arrière-fond de paysage terrestre d'hiver à teneur fortement symbolique. Nous sommes donc loin des illustrations plus ou moins artistiques de rigueur dans les collections jeunesse. Ajoutons à cela la mise en page: plus de 425 pages, contrairement à la moyenne des $125-180$ pages de ces mêmes collections; le nom de l'éditeur, Québec/Amérique, et non plus Québec/Amérique Jeunesse; le résumé, en quatrième de couverture, insistant sur l'enjeu bien adulte du conflit romanesque ${ }^{7}$; la note biographique, franchement commerciales; la séquence d'extraits de critiques dithyrambiques et, finalement, à l'intérieur, à côté de la page de titre, l'impressionnante bibliographie axée sur la division "Pour les jeunes/Pour les adultes" ( $L P$, p. 4). Tout le support matériel convie exclusivement les adultes à la lecture.

Mais qu'en est-il de l'écriture du Pari? S'énonce-t-elle en soi conformément à une prétendue spécificité "pour adultes seulement"? Regardons le prologue:

La digue a tenu pendant de longues années au cours desquelles mes souvenirs d'enfance semblaient endormis. Parfois au hasard d'une rencontre, d'un mot ou d'un bouquet d'odeurs, le

7. "Deux médecins prennent un pari terrible [...] Max Laforest, une omnipraticienne de 40 ans, et Ala, sa mystérieuse pariente. Elles ont tout fait pour fuir leur passé, elles doivent maintenant l'affronter ( $L P$, quatrième de couverture).

8. "Après le retentissant succès de Maina (Québec/Amérique, 1997), titré longtemps sur la liste des best-sellers, et de Marie-Tempête (Québec/Amérique, 1997) qui jouit maintenant d'un rare succès en France et dont on a tiré un téléfilm, Dominique Demers nous propose son nouveau roman pour adultes* ( $L P$, quatrième de couverture). 
mur se lézardait et le passé ruisselait. Je renouais avec l'horreur ou l'enchantement. De la masse confuse des années emmêlées émergeaient tour à tour des pommiers en fleurs et des spectres effrayants.

Je sais à quel moment la digue s'est rompue. J'arrive à déterminer le jour, le point tournant, mais je minterroge encore sur l'enchaînement des moments décisifs ( $L P$, p. 15$)$.

Le temps s'impose ici comme le fondement de l'ouvre littéraire pour le lecteur adulte: moins à cause de l'évidence biologique, l'âge de la narratrice, que grâce à la polyvalence du point de vue que permet la crise de la maturité. Certes, le retour dans le temps peut exister aussi dans certaines cuvres pour la jeunesse, mais à la condition expresse de faire disparaître très rapidement le narrateur de l'âge mûr ${ }^{9}$; en revanche, dans les ouvres pour adultes, le retour sur soi du narrateur permet de substituer à la linéarité typique des romans pour la jeunesse la circularité et la réversibilité. Dans les jeux temporels du Pari, le présent explique et modifie le passé comme le passé informe et déconstruit le présent: Maximilienne Laforest devient une conscience dans le temps, un je multiple et complexe inscrit dans un réseau d'êtres également complexes, modifiables, à l'opposé de Marie-Lune qui, malgré les petits récits rétrospectifs, vit son difficile passage à l'âge adulte dans un "ici et maintenant" progressif. Pour faire du Pari une ceuvre pour la jeunesse, il faudrait sans doute un reformatage, voire une réécriture, ce qui rêlève pour l'instant de la spéculation.

L'orientation inverse, soit la réédition pour adultes d'un roman pour la jeunesse, peut s'avérer plus retorse. En ce qui concerne Dominique Demers, deux cas: la métamorphose de a MarieLune" en "Marie-Tempête", et la publication quasi simultanée de Maina pour deux publics. Le premier, Marie-Tempête, semble moins évident ou prémédité a priori, puisqu'il y a eu changement d'éditeur (de La courte échelle à Québec/Amérique Jeunesse), travail d'écriture dans le temps (1992-1994), et recentrage de la série comme le suggère la quatrième de couverture: "Elle

9. Entre autres exemples, le roman de François Gravcl, Kate, quelque part (Montréal, Québec/Amérique, coll. "Titan +", 1998), est particulièrement significatif à cet égard. Un narrateur d'âge mûr de la fin des années $1990 \mathrm{se}$ souvient de sa première expérience amoureuse dans les années 1960: or, dès le second chapitre, l'instance énonciative perd de vue l'âge du narrateur, si bien que, progressivement, les temps du passé font place au présent de la narration, qui domine jusqu'à la clôture du récit. 
s'appelle Marie-Lune, mais on dirait plutôt une Marie-Tempête ${ }^{10}$ ". L'unité sérielle est devenue plus éclatante après la reprise en main par Québec/Amérique, d'autant plus que le tome I conservait un degré d'autonomie narrative malgré certaines pistes, ou amorces de récits, que nous verrons plus tard.

Dans le second cas, la transition de Maïna I à Maïna II relève moins de la sériation que de la tomaison, le découpage en deux volumes paraissant attribuable autant à la logique du récit qu'au format de la collection, comme on l'observe fréquemment chez Michel Quintin, éditeur spécialisé dans ce qu'on pourrait appeler le genre "roman d'aventures hommage aux Premières Nations ${ }^{11}$. Et pourtant, le discours préfaciel cible les destinataires: "À tous les jeunes qui aiment la lecture, les découvertes, l'amour, l'aventure... J'ai écrit ce roman pour vous" ( $A L$, p. 11), clarification supprimée dans la reprise de la dédicace pour adultes.

Compte tenu de la parution presque simultanée de Maina pour grand public, faut-il accorder un crédit absolu à cette profession de foi de l'auteure, sans doute sensible aux pressions de l'institution littéraire, c'est-à-dire au prestige qu'apporte la publication pour adultes? Ou doit-on n'y voir que la stratégie d'un éditeur cherchant à maximiser les profits d'un produit culturel, certes rentable, mais "ghettoïsé ${ }^{12}$ "? Pour reprendre un paradoxe emprunté aux dissertations d'antan, si la forme crée le fond, le format conditionne l'effet littéraire et dicte la perception de l'œuvre.

\section{La hiérarchisation des effets de style}

Il serait ici essentiel de comparer in toto les versions de "Marie-Lune "/a Marie-Tempête" afin de repérer toute variante indicatrice d'un quelconque travail de réécriture, ce qui légitimerait le

10. "Marie-Lune I", réédition en 1998 d'Un biver de tourmente, ouvr. cité.

11. Chez cet éditeur (Waterloo/Québec, Éditions Michel Quintin, coll. "Grande Nature "), signalons de Michel Noël, Dompter l'enfant sauvage, en deux volumes (t. 1: Nipisbish, t. 2: Le pensionnat, 1998) et d'André Vacher, Le vieil Inuk, également en deux volumes (t. 1: Le loup blanc; t. 2: La statuette magique, 1999).

12. Sur la motivation de Dominique Demers et les stratégies de son éditeur, voir l'article très informé bien que bref: Daniela Di Cecco, "Maïna et MarieTempête: deux romans, deux publics", Canadian Children's Literature/Littérature canadienne pour la jeunesse (CCL/LCJ), Guelph (Ontario), vol. 26, $\mathrm{n}^{n} 1$, printemps 2000 , p. $27-30$. 
fait de parler d'ouvrages distincts: "Marie-Tempête", ainsi, ne serait plus "Marie-Lune". Or, à première vue, il s'agirait plutôt, dans les deux cas, d'une réédition, avec mise en page ou reformatage à l'intention des seuls adultes, de tous les adultes, et non plus des parents curieux des lectures de leur progéniture et du jeune public-limite consommant indistinctement romans pour ados et romans pour adultes. Supposons que le texte narratif des deux versions, à l'exclusion du paratexte, soit identique; il devient alors loisible de nous demander si la dichotomie entre "littérature" et "littérature pour la jeunesse " est une simple différence de degré ou bien, au sens pascalien du terme, une différence de nature ou d'ordre. Autrement dit, lorsqu'on passe de l'une à l'autre, dans les cas concrets qui se multiplient dans la production québécoise actuelle, est-ce d'abord une question de réécriture, de stylométrie (longueur des phrases, lisibilité lexicale, etc.), de closage des effets littéraires, ou est-ce un faux problème, le lecteur (par choix personnel) ou l'éditeur (par politique ou par calcul de marketing) lui conférant la légitimité culturelle "i? Ces interrogations sont d'autant moins gratuites que Dominique Demers elle-même, à la fois spécialiste et praticienne de la littérature pour la jeunesse, s'est attachée à en définir la spécificité ${ }^{1 i}$.

À la lumière de ces observations, comparons les incipits

\section{- d'Un biver de tourmente:}

Ma mère a les cheveux bleus. Elle n'est pas complètement marteau, ni même un peu Martienne, mais simplement coloriste au Salon Charmante, rue Principale à Saint-Jovite. La semaine dernière, ses cheveux étaient "or cuivré ". Le flacon 57, sur l'étagère du hatut.

Derrière les séchoirs, tout au fond du salon, ma mère mélange des couleurs. Mèches, teintures, balayages, reflets... Il y a des peintres en bâtiment, dautres en chevelure. [...]

13. Voir à ce propos la série d'articles de jean-François Boutin sur * Le problème du corpus de rextes littéraires en classe de langue première: "Examen des idées de littérature et de littérature denfance et de jeunesse", CCL/LCI, vol. 24, no 3/4, automne/1hiver 1998, p. 83-102; "Entrevuc avec 32 agents des champs littéraire et scolaire en regard des idées de littérante et de littérature d'enfance et de jeunessé, vol. 25. n" 2, été 1999, p. 42-59; "Itinéraire pour une ouverture des corpus au primaire et au secondaire "vol. $25, \mathrm{n}^{\circ} 4$, hiver 1999 , p. 37-61.

14. Voir Dominique Demers, avec la collahoration de Paul Bleton, Du Petit Poucet an Dernier des raisins. Introduction à la littérature jeunesse, Montréal, Québec/Annérique Jeunesse et Télé-université, 1994. 
Moi, je rêve d'une mèche bleu électrique. Juste une, presque discrète, qui se tiendrait bravement debout sur le dessus de ma tête. Mais pas question! La petite Marie-Lune de Fernande et Léandre n'a pas le droit d'être punk. Je me contente d'une coupe légèrement étagée et terriblement ordinaire, signée Gaëtanne, propriétaire du Salon Charmante ( $H T$, p. 11-12).

- de Maina:

Maïna voulait tuer. Planter sa lance et voir mourir avant qu'il fasse brun. Tuer, puis éventrer, éviscérer, écorcher et porter la bête encore chaude jusquau camp. Elle avançait à grands pas souples mue par ce désir immense qui l'habitait tout entière. Lá veille, des hommes avaient ramené un caribou que l'hiver navait pas trop amaigri. Malgré sa grande faim, Maïnáa avait détourné son regard des entrailles fumantes. Le chef, Mishtenapeu, avait compris que sa fille renonçait à la nourriture afin d'anadouer les esprits avant d'accomplir un geste sacré. Maïna espérait qu'en échange le Manitou lui livrerait une bête ( $A L$, p. 17).

D'une part, un énoncé aux résonances éluardiennes, de lautre, une attaque de roman d'action: deux phrases-chocs, brèves, incisives, lapidaires. Mais les divergences vont bientôt l'emporter sur les similitudes. Du point de vue stylistique, pour "Marie-Lune", le maintien de la phrase courte, "punchée" du format pour adolescents, et toujours le fameux ton direct de Dominique Demers; à l'opposé, pour Maina, un élargissement vers la phrase complexe. Quant à la thématique de ces incipits, une affirmation de soi fondamentale et une quête d'identité en gestation chez les deux héroïnes. À l'éclosion du conflit mère/fille répond le désir de transgresser le clivage entre les sexes, qui risque d'entraîner l'exclusion du clan. Bref, malgré un éloignement temporel vertigineux, se coiffer à la punkette et bouleverser, au néolithique, la répartition des rôles relèvent du même fond de commerce des romans pour adolescents : le conflit entre générations.

Mais la différence se creuse de plus en plus rapidement, du seul fait, entre autres facteurs narratifs, du passage de la narration homodiégétique à la narration hétérodiégétique. Pour Un biver de tourmente, la narration à la première personne rappelle les romans humoristiques pour les huit-douze ans de la romancière, mais, cette fois-ci, malgré l'humour, il y a irruption d'un drame intime (c'est dans la dérision qu'est consommée la rupture d'avec la mère); de même, les traits culturels propres à l'adolescence s'inscrivent dans un "ici et maintenant" qui disqualifie la génération des parents. Enfin, les rapprochements stylistiques 
"marteau/martienne", "peintre en bâtiments/peintre en chevelure", autrement dit l'accumulation des marques dépréciatives, donnent un ton de modernité littéraire, typique de l'après-Dernier des raisins ${ }^{15}$. Ce ton communique aux adolescents l'impression d'être en présence de quelqu'un qui parle leur langage, et aux adultes, un effet d'authenticité et de "vécu. enregistré sur le vif.

Quant à Maina, la prise en charge par une instance narrative extérieure permet le passage entre les voix des personnages: le refus de la jeune fille est deviné par le père mais interprété, décrypté par la voix narrative qui, ce faisant, assume une posture anthropologique. Le narrateur prend en charge la compréhension du père et instruit ses narrataires, d'où un investissement pédagogique. Ce changement de perspective, du personnage de Maïna à celui du père et à l'instance anthropologique, recoupe l'effet des éléments stylistiques: la synonymie et la recherche lexicale ainsi que la variation dans les structures syntaxiques fonctionnent

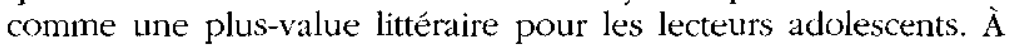
la découverte et à l'approfondissement du monde du personnage se surajoute l'enrichissement langagier et culturel du jeune lectorat. On pressent, malgré la facilité d'un tel rapprochement, que "Marie-Lune" fait plus littéraire au sens moderne du terme - la révolution des années 1980 en littérature jeunesse du Québec correspondant à la révolution romanesque des années 1960 tandis que Maïna fait plus littéraire au sens conventionnel, "académique", du terme.

\section{La métaphore comme marqueur culturel}

Pour s'en convaincre davantage, il suffit de considérer l'usage et le registre des métaphores. En premier lieu, dans Un biver de tourmente:

Je me suis réveillée ce matin avec un bébé bouton éléphantesque sous le nez. Les boutons, c'est comme les embryons. Ça enfle tranquillement. Au début, notre face ne sait pas qu'elle est enceinte. Dans mon cas, l'accouchement ne devrait pas tarder.

15. Rappelons que, pour Dominique Demers, "[cle roman a profondément marqué la littérature jeunesse québécoise et a contribué à moderniser l'écriture romanesque du récit pour adolescents" (Dominique Demers, Du Petit Poucet au Dernier des raisins, ouvr. cité, p. 230 et suiv,). 
Il est arrivé quelque chose d'étrange ce matin. Fernande avait la voix rauque, les yeux pochés, le visage pâle et les gestes lourds. On aurait dit que tous les membres pesaient trois tonnes. Elle a encore téléphoné à Gaëtanne pour annoncer qu'elle serait en retard. Ma mère sirotait lentement son café quand uné mésange a piqué dans la fenêtre de la cuisine.

Fernande s'est mise à hurler comme un chien blessé en voyant le petit paquet de plumes inerte. Rien ne semblait pouvoir l'arrêter. Léandre l'a entraînée vers la chambre à coucher. (HT, p. 31-32)

L'assimilation de l'acné à l'embryon prolonge la thématique de la révolte et du mal de vivre, mais agit a posteriori en tant qu'annonce du second tome (la grossesse de l'héroïne); les clichés du vieillissement de la mère tiennent à la fois du magazine féminin et de l'exagération oralisante des adolescents ("trois tonnes"); le présage (l'oiseau de malheur) ressortit autant à la superstition populaire qu'à l'état réel de la mère: le contraste entre la fragilité de la mésange et la réaction hystérique du personnage préfigure le drame à venir (le cancer de Fernande). Même la remarque sur l'indolence de la coiffeuse - elle est signe de paresse chez l'adulte, mais marque de créativité et d'affirmation de soi chez l'adolescente - renforce la cohérence du point de vue de la narratrice. En apparence, ce sont des procédés faciles, mais ils s'avèrent d'une indéniable efficacité. Le lecteur, adolescent ou adulte, trouvera ce discours et cet univers culturel crédibles chez une adolescente de quinze ou seize ans.

En ce qui concerne Maïna, c'est une tout autre histoire. En particulier, l'expression amenant le transfert métaphorique, "on aurait dit ", conditionnel passé première forme, normal et acceptable dans le discours d'une adolescente première de classe, se transforme en un conditionnel passé deuxième forme, "on eût dit ", forme savante qui renvoie à la littérature à l'ancienne, celle de l'écriture bourgeoise, pour emprunter une formule bourdieusienne ${ }^{16}$.

Le soleil reprit des forces alors que celles des Presque Loups déclinaient de nouveau. La neige devint brillante, comme hérissée des flèches qui brûlaient les yeux. Un matin, de larges

16. Il n'est que trop tentant de rapprocher lè style à la Rosny Aîné de Maina aux tableaux des "peintres pompiers" de la seconde moitié du XIX" siècle. Voir Pierre Bourdieu, Les règles de lart. Genèse et structure du champ littéraire [1992], Paris, Seuil, coll. "Points*, 1998, p. 185. 
volées d'outardes obscurcirent le ciel. La glace fendit, craqua, et creva, libérant l'eau. On eût dit une bête éventrée dont les entrailles noires surgissent. D'instinct, les Presque Loups repartirent vers la côte, un triste cortège d'hommes, de femmes et d'enfants, épuisés, amaigris, malades et affamés, glissa silencieusement sur la rivière aux loutres. Maina fouilla souvent le paysage dans l'espoir d'apercevoir la bande de Manutabi. Mais ils approchèrent de la dernière chute sans avoir rencontré d'autres Presque Loups. Maïna fut prise d'épouvante à l'idée que Manutabi ne reviendrait peut-être pas. Elle continua à pagayer le cour écrasé par les serres d'un aigle géant ( $A L$, p. 99).

Bonjour les métaphores! ne peut-on s'empêcher de souligner en se rappelant les préventions d'Alain Robbe-Grillet ${ }^{17}$. Concédons néanmoins un degré élevé de pertinence à celles-ci: les forces antithétiques du soleil et des Amérindiens, les flèches de la lumière, la glace s'ouvrant comme une bête éventrée renvoient bien à l'univers animiste, à la fois anthropomorphique et sacralisé, dans lequel évolue Maïna. Ces métaphores s'avèrent autant d'indices de mythes, d'échanges entre l'univers des humains et celui des esprits. Et pourtant, on ne peut s'empêcher de percevoir, clans ces effets stylistiques à la Félix-Antoine Savard, les accessoires d'un musée Grévin de la belle et grande littérature d'autrefois. Pour le jeune lecteur, c'est l'accession à la littérature bourgeoise; pour un lecteur adulte, c'est la marque d'une ouvre bien écrite, de la qualité littéraire.

La cohésion thématique de l'œuvre romanesque de Dominique Demers demeure impressionnante, mais on ne saurait ignorer la présence d'indicateurs de niveaux d'âge ou de groupes culturels de lecteurs, tels le choix de la voix narrative, le passage de l'humour au drame, le transfert des registres stylistiques, etc., qu'il serait possible de répertorier. Cependant, pour bien cerner la différence entre la "littérature" et la "littérature pour la jeunesse", comme chez tous les auteurs d'ailleurs, il ne saurait y avoir de meilleur repère que le traitement de la sexualité. À la litote généralisée des collections pour adolescents s'oppose le dit, la franchise ou la surenchère de la littérature pour adultes ${ }^{8}$. Au reste, la

17. "La métaphore, rappelait Robbe-Grillet, n'est jamais une figure innocente": elle finit par "révéler tout un système métaphysique". Voir Alain Robbe-Grillet, Pour un nouveau roman [1963], Paris, Gallimard, coll. "Idées", 1968, p. 60.

18. Chez Dominique Denmers, bien quil existe une pudeur certaine quant à l'expression de la sexualité, on peut observer une diffétence de degré per 
frontière entre littérature pour la jeunesse et littérature pour adultes demeure souvent ténue, à moins que la censure ne vienne servir de révélateur de cette frontière. Maina, par exemple, a valu des ennuis à Dominique Demers, la publication de la version pour tous publics incitant sans doute les parents à regarder de plus près la version pour adolescents mise au programme scolaire et distribuée aux élèves ${ }^{19}$.

En conclusion, on pourra songer à quel point la préface de Jacques Allard à Marie-Tempête prend tout son sens dans ce contexte. Ce qui compte, lorsque ce critique établi, pilier de l'institution littéraire, pose la question "y a-t-il vraiment des écrivains "jeunesse"? N'y a-t-il pas plutôt des écrivains, point?» (MT, p. 9), c'est moins l'énoncé lui-même que l'acte d'énonciation et la posture de l'énonciateur. L'œeuvre est dédouanée et le lecteur adulte est absous, rédimé: il peut lire l'excellent ouvrage pour adolescents de douze ans et plus sans mauvaise conscience, comme un vrai livre, et sans redouter le regard désapprobateur de ceux qui tolèrent mal qu'un être censément parvenu à maturité consomme un produit au-dessous de son âge.

ceptible dans la scène de défloration de l'héroïne. Que l'on se reporte à la p. 102 d'Un biver de tourmente et à la p. 238 du Pari pour constater le passage de la métaphore filée (la nuit sur une mer agitée, $H T$, p. 102) à l'indication de détails concrets: "[s]on sexe était gonflé et dur sous mon ventre, etc. " (LP, p. 238-241).

19. Le cas de Marie Laflamme (Paris, Denoël/Jai lu, 3 vol., 1995) de Chrystine Brouillet, célèbre et prolifique romancière produisant pour tous les publics, est à cet égard emblématique: la fameuse cabale des parents du Collège Brêbeuf met en relief le ciblage d'une ceuvre pour un public autre que celui d'origine. Il a suffi qu'un roman pour adultes, somme toute assez bénin, soit mis entre les mains d'élèves de bonne famille du secondaire pour qu'il devienne subversif et corrupteur, et pour qu'un bref paragraphe, une scène de nécrophilie plutôt furtive, se transforme en représentation du Mal absolu. 